\title{
Fabrication of Columnar Sub-microstructures using a Q-switched Nd:YAG Laser in the Nanosecond Time Regime
}

\author{
Jorge Castellanos, Samuel A. Betancourt-Pagan, Edgardo J. Robledo-Ortiz, Leonardo C. Pacheco-Londoño, \\ Marco A. De Jesús and Samuel P. Hernández-Rivera \\ Chemical Imaging Center, ALERT-DHS Center of Excellence for Explosives Research \\ Department of Chemistry, University of Puerto Rico-Mayagüez, Mayagüez, PR 00681 \\ E-mail: jorge.castellanos1@upr.edu,samuel.hernández3@upr.edu
}

\begin{abstract}
We report here on the formation of columnar sub-microstructures in single-side polished $<100>$ silicon wafers by optimizing ablation laser parameters. Laser ablation was performed in air and the parameters, fluence and the number of pulses of the third harmonic of a Q-switched Nd:YAG laser operated at fixed repetition rate of $10 \mathrm{~Hz}$ were investigated. Columnar sub-microstructures were predominantly observed along the border of the ablation centers in the silicon substrates that were placed $9.3 \mathrm{~cm}$ in front of a $10 \mathrm{~cm}$ focal length quartz lens. Silicon substrates were exposed to fluence of 1.0 and $1.5 \mathrm{~J} / \mathrm{cm}^{2}$ and 50-2000 laser pulses. Columnar sub-microstructures at the ablation centers were observed only for 50 and 100 pulses at $1.0 \mathrm{~J} / \mathrm{cm}^{2}$. The average period of these structures, with the parameters optimized, was close to the wavelength, reaching heights in the hundreds of nanometers to slightly greater than $1 \mu \mathrm{m}$ above the surface. Columnar formation was non-existent at lower fluence of 0.15 and $0.3 \mathrm{~J} / \mathrm{cm}^{2}$, which were characterized by laser-induced periodic surface structures.
\end{abstract}

DOI: $10.2961 /$ jlmn.2015.03.0005

Keywords: laser ablation, silicon substrates, Q-switched Nd:YAG, nanosecond pulses, laser fluence

\section{Introduction}

Laser ablation of substrates in the nano-, pico- and femtosecond time regimes tends to produce spontaneous self-assembled micro/nano conical or columnar shaped structures that can be prepared and optimized by controlling laser parameters [1-4]. The technique offers an alternative to more costly and complex lithographic and chemical etching routes [1]. Applications can be found in photovoltaics (e.g., solar cells), photodetectors and other sensing technologies such as in surface enhanced Raman scattering (SERS), medical devices and laser desorption ionization (LDI) of biomolecules [2-7]. In order to fabricate these structures, special attention must be given to the number of incident laser pulses, beam diameter, laser fluence $\left(\mathrm{J} / \mathrm{cm}^{2}\right)$, laser wavelength, pulse duration and ablating environment $[7,8]$. At high laser fluence values in the nanosecond (ns) regime, the shock dynamics observed at the laser beamablated material surface interface is characterized by shockwaves that lead to crater formation $[9,10]$. Below the conical/columnar formation fluence range $\left(1\right.$ and $\left.10 \mathrm{~J} / \mathrm{cm}^{2}\right)$, ripple patterns or laser-induced periodic surface structures (LIPSS) with periods near to that of the irradiating wavelength are observed between 0.1 and $1 \mathrm{~J} / \mathrm{cm}^{2}[7,11,12]$. These patterns are observed just above the melting threshold of the material that forms from the interference of the incident laser light with scattered light near the substrateablating media interface $[8,11,13,14]$. In addition, the cones or columnar shaped structures align along the laser beam axis and their heights are typically at or above the substrate $[3,12,15]$.

Structures formed using water as ablating medium were roughly an order of magnitude smaller in height and width than cones formed in a gaseous environment $[7,16]$. Increasing the number of pulses also increased their heights with little effect on their transversal dimension [15]. As long as the laser fluence criterion was met, large area coverage was attained without the use of a focusing lens [11]. However, fluence at larger areas requires higher energy pulses, which may be difficult to achieve at shorter wavelengths when using a harmonic generator because of successive losses of power when mixing frequencies.

The objective of this work was to optimize the fabrication of columnar sub-microstructures using photonic ablation with a Q-switched laser operating in the nanosecond regime and varying the parameters that control the process. For practical reasons we have defined optimization where the resulting period/spacing of these structures was smallest. The effects of laser beam diameter, fluence and number of pulses in air were investigated at the third harmonic of a nanosecond Nd:YAG laser. The beam diameter was determined using a knife-edge method and power/energy sensor combination. Texturing was performed at low fluence $(0.15$ $\mathrm{J} / \mathrm{cm}^{2}$ and $\left.0.30 \mathrm{~J} / \mathrm{cm}^{2}\right)$ and high fluence $\left(1.0\right.$ and $\left.1.5 \mathrm{~J} / \mathrm{cm}^{2}\right)$. Optimization was observed at $1.0 \mathrm{~J} / \mathrm{cm}^{2}$ and 50 pulses.

\section{Experimental}

Undoped, single-side polished, $<100>$ silicon $(\mathrm{Si})$ round wafers ( $\mathrm{R}>1000 \mathrm{ohm}-\mathrm{cm}$ ), $25.4 \mathrm{~mm}$ in diameter (MTI Corp., Richmond, CA, USA) were used for the experiments. Surfaces were ablated with $3^{\text {rd }}$ harmonic pulses ( 355 $\mathrm{nm})$ generated with a non-linear crystal of a flash-pumped Quanta-Ray PRO 290 Nd:YAG laser (Spectra-Physics, Newport Corp., Irvine, CA, USA). The laser was operated at a fixed repetition rate of $10 \mathrm{~Hz}$. Gaussian laser pulses generated by operating in Q-switch mode (7.9 ns pulse width) were characterized using a knife-edge method guide and manipulate the laser beam. A mirror and $10 \mathrm{~cm}$ quartz lens $(10 \mathrm{~cm}$ focus) was used to manipulate the laser beam. 


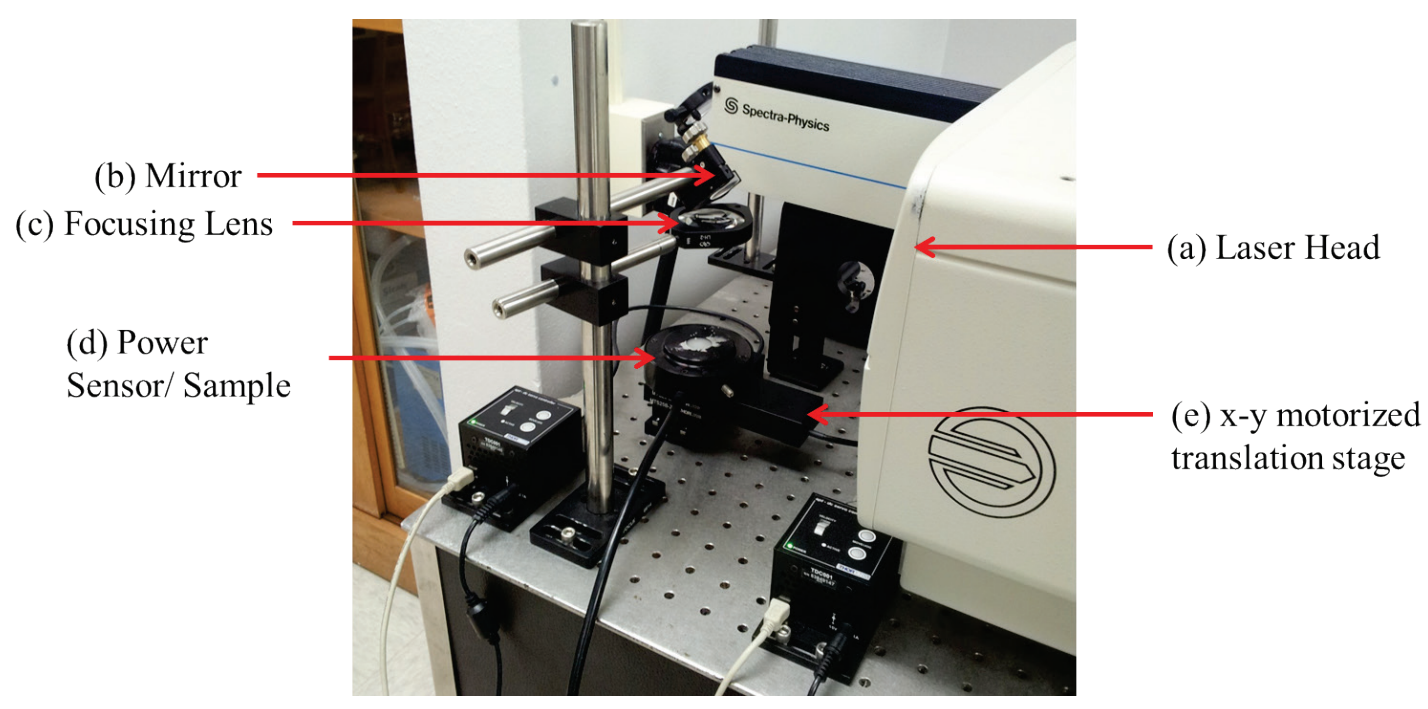

Fig. 1 Experimental setup for the ablation of Si wafers and determination of the laser beam diameter at the surface. The beam propagated from the laser head (a), reflected off a mirror (b) and passed through a quartz focusing lens (c) before arriving at the sensor (d) on an $x-y$ translation stage (e). For ablation of silicon experiments, the same setup was used except the power meter was replaced by a Si wafer.

To minimize damage to the power/energy sensor, the beam was profiled at $7 \mathrm{~mm}$ from the plasma (measured with a caliper). Briefly, a Nova II power/energy sensor (OphirSpiricon, LLC North Logan, UT, USA) placed below a razor blade was mounted on a motorized $x-y$ translation stage with a $29 \mathrm{~nm}$ travel resolution (Thorlabs, Inc., Newton, NJ, USA) and rastered at $20 \mu \mathrm{m} / \mathrm{s}$. The power of the train of pulses was measured until reaching a threshold value of $\sim 0.120 \mathrm{~W}$ before normalization [17-21]. The laser power data collected by the meter were stored using StarCom32 software, v3.2 (Ophir-Spiricon) interfaced to via a RS232 cable. The data were smoothed in OriginPro ${ }^{\mathrm{TM}}$ v8.1 SR0 (OriginLab Corp. Northampton, MA, USA) using the Savitzky-Golay filter [22, 23]. A polynomial regression using a window of 20 points was performed at each data point and its smoothed value was determined. The smoothed data were regressed for ten separate trials and the average beam diameter as per full width at half maximum (FWHM) was determined. For the ablation experiments of $\mathrm{Si}$ wafers, the Gaussian beam emitted at the laser head was reflected with a mirror and passed through the focusing lens. However, the beam arrived at the $\mathrm{Si}$ wafer instead of the power sensor and the $\mathrm{x}-\mathrm{y}$ translation stage was kept stagnant. The setup is shown in Fig 1. For texturing experiments in air, fluence values of $0.15,0.31 .0$ and $1.5 \mathrm{~J} / \mathrm{cm}^{2}$ were used at a number of pulses of $50-2000$ at $7 \mathrm{~mm}$ in front of the focal point of the lens. Furthermore, texturing of the Si substrates was explored at the focal point for a fluence of $3.0 \mathrm{~J} / \mathrm{cm}^{2}$ using the same range of pulses. Scanning electron microscope (SEM) micrographs were collected using a JEOL model JSM-6360 (Peabody, MA, USA). The tip width, column width and period were measured using Image $\mathrm{J}^{\mathrm{TM}} \mathrm{v} 1.48$ image processing and analysis software (National Institutes of Health, NIH, Bethesda, MD, USA) using a pixel to distance relationship established with the scale bars of the SEM micrographs. Surface morphology characterization measurements were performed using an Alpha Step IQ stylus-based surface profiler (KLA-Tencor Corp., Milpitas, CA, USA). Finally, atomic force microscope (AFM) images were collected in semi-contact mode using a scanning probe microscope system model NTEGRA Spectra SPM (NT-MDT, Tempe, AZ, USA).

\section{Results and Discussion}

One of the most important parameters to consider when controlling the formation of columnar sub-microstructures on Si substrates upon laser ablation is the laser spot diameter, used to calculate the laser fluence [24]. Laser fluences between 1.0 and $5.0 \mathrm{~J} / \mathrm{cm}^{2}$ have produced microstructures on a variety of materials, including $\mathrm{Si}[13,15,25]$.

The beam width at FWHM at $7 \mathrm{~mm}$ from the focal point was calculated as $1.10 \mathrm{~mm}$ using $\mathrm{w}_{\mathrm{o}}=934 \mu \mathrm{m}$ as determined by the fit using Equation 1:

$$
P=P_{\mathrm{o}} e^{\left(-\frac{2 r^{2}}{w_{o}^{2}}\right)}
$$

where $\mathrm{P}$ is the laser beam power at the surface, $\mathrm{P}_{\mathrm{o}}$ is the unobstructed peak power, $\mathrm{r}$ is the radial position in micrometers and $\mathrm{w}_{\mathrm{o}}$ is the Gaussian beam radius at $1 / \mathrm{e}^{2}$ of the peak power.

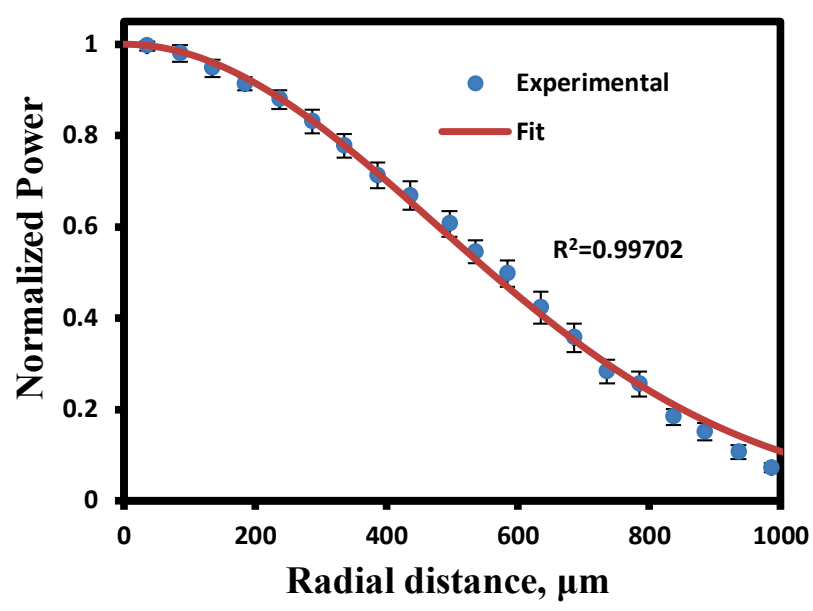

Fig. 2 Gaussian beam profile of the $355 \mathrm{~nm} 10 \mathrm{~Hz}$ ns pulses measured approximately $7 \mathrm{~mm}$ in front of the focal point. Error bars denote the standard deviations of the smoothed data for ten trials. 


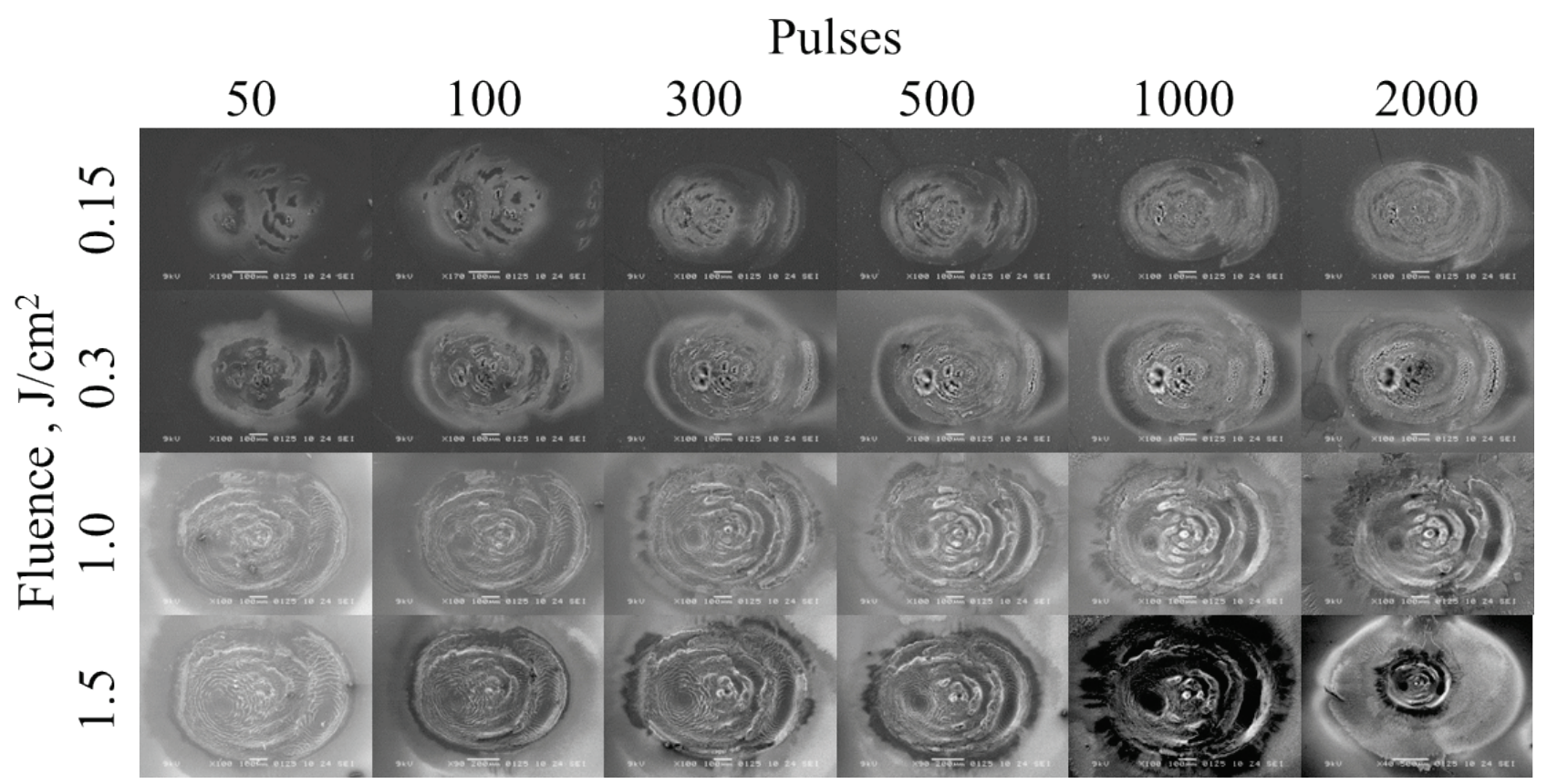

Fig. $3 \mathrm{Si}$ surface ablations using the third harmonic of the Nd:YAG nanosecond laser at fluence of $0.15-1.5 \mathrm{~J} / \mathrm{cm}^{2}$ and $50-2000 \mathrm{pulses}$.

Therefore, the Gaussian beam diameter at FWHM was determined as $1.18 \mathrm{w}_{\mathrm{o}}$ (Fig. 2) [26]. Several texturing regions can be observed at 1.0 and $1.5 \mathrm{~J} / \mathrm{cm}^{2}$ at all number of pulses. These include craters, roughened and smoothed surfaces at the ablation centers consistent with repetitive nanosecond pulsed irradiation (Fig. 3). Furthermore, a surface profile of the crater obtained at $1.0 \mathrm{~J} / \mathrm{cm}^{2}$ at 50 pulses shows uneven ablation across the irradiated spot resulting in ridges possibly formed from inhomogeneities in the laser beam (Fig. 4). Column formation is mostly apparent at the border lining the ablation center for $1.0 \mathrm{~J} / \mathrm{cm}^{2}$ and $1.5 \mathrm{~J} / \mathrm{cm}^{2}$ (Fig. 5). Nayak et al. reported similar observations at the edge as an annular region of nanoprotrusions [2]. In our work, increasing the fluence and number of pulses had the effect of narrowing the columnar sub-microstructures borders, enlarging the base and flattening the columns to produce bubble shaped structures possibly caused by a remelting of Si at the site (Fig. 5a-f). There was no considerable roughening observed at $0.15 \mathrm{~J} / \mathrm{cm}^{2}$ or at $0.30 \mathrm{~J} / \mathrm{cm}^{2}$ on $\mathrm{Si}$.

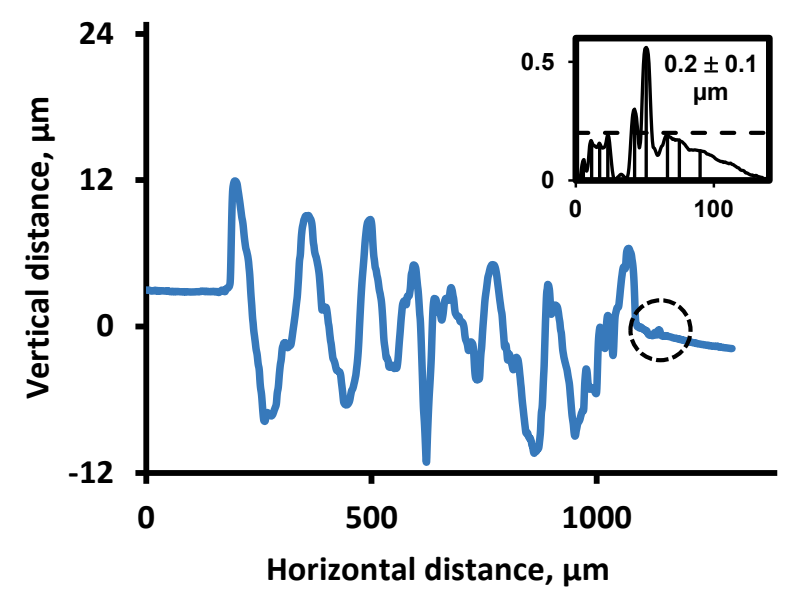

Fig. 4 Surface profile of crater formed at $1.0 \mathrm{~J} / \mathrm{cm}^{2}$ and 50 laser pulses. Inset: zoom of height profile of the region marked by circle.
Instead, laser-induced periodic surface structures (LIPSS) were evident and pronounced with increasing number of pulses at these low fluence regimes. A closer look into the columnar sub-microstructures revealed high periodicity at low \# of pulses (50 and 100$)$ for $1.0 \mathrm{~J} / \mathrm{cm}^{2}$ and deformities developing at higher \# of pulses $(300-2000)$ (Fig. 6a-e). Low values of $\#$ of pulses $(50-100)$ proved to be optimum for fluence of $1.0 \mathrm{~J} / \mathrm{cm}^{2}$ where the highest structure density was observed.

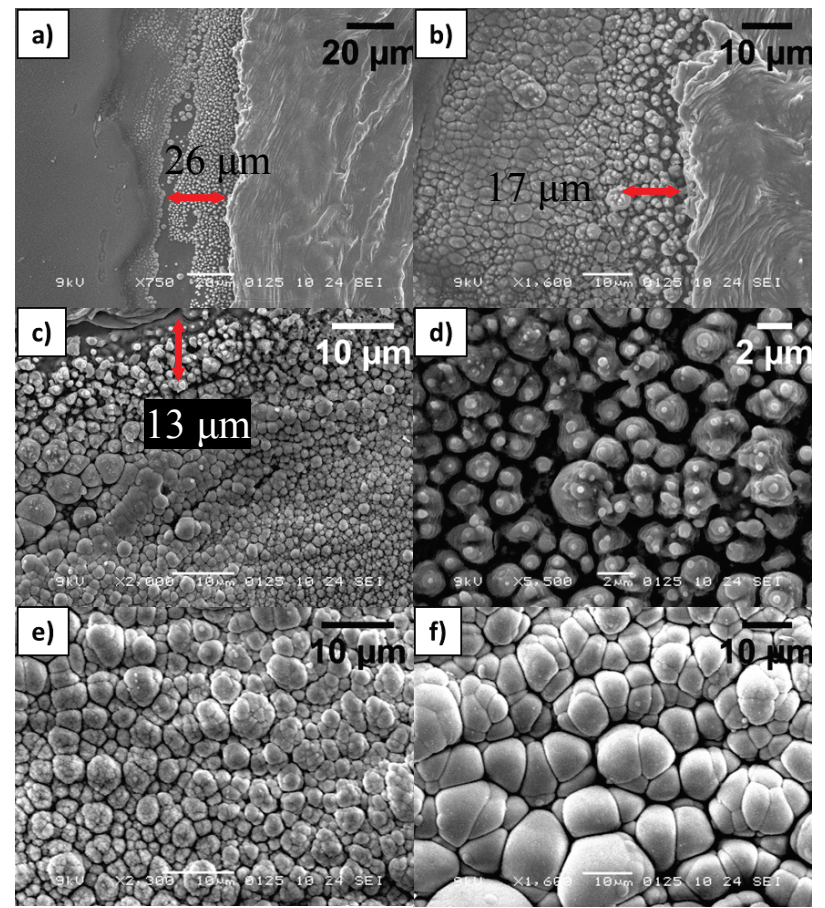

Fig. 5 Microtexturing of Si with varying fluence and number of pulses bordering the ablation center. a) $1.0 \mathrm{~J} / \mathrm{cm}^{2}$ at 100 pulses (scale bar: $20 \mu \mathrm{m}$ ); b) $1.0 \mathrm{~J} / \mathrm{cm}^{2}$ at 300 pulses (scale bar $10 \mu \mathrm{m}$ ); c) $1.5 \mathrm{~J} / \mathrm{cm}^{2}$ at 300 pulses (scale bar $10 \mu \mathrm{m}$ ); d) $1.5 \mathrm{~J} / \mathrm{cm}^{2}$ at 300 pulses (scale bar $2 \mu \mathrm{m}$ ); e) $1.5 \mathrm{~J} / \mathrm{cm}^{2}$ at 1000 pulses (scale bar $10 \mu \mathrm{m})$; f) $1.5 \mathrm{~J} / \mathrm{cm}^{2}$ at 2000 pulses (scale bar $10 \mu \mathrm{m}$ ). 


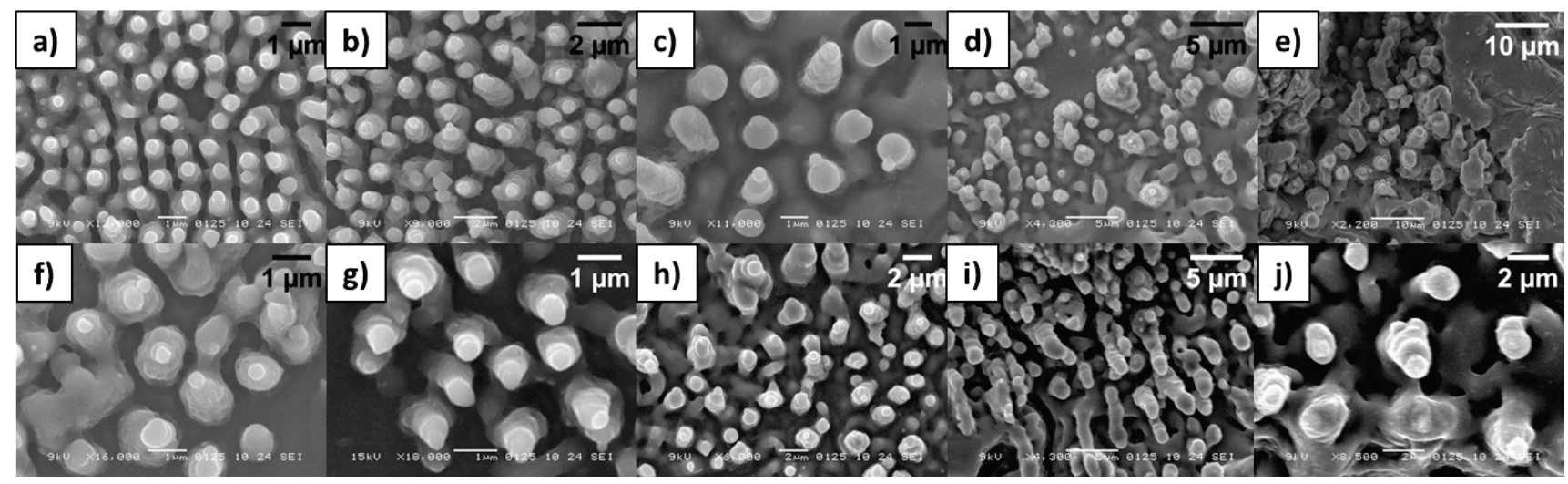

Fig. 6 Columnar microstructures bordering ablation centers on Si for fluence $1.0 \mathrm{~J} / \mathrm{cm}^{2}$ (top row) and $1.5 \mathrm{~J} / \mathrm{cm}^{2}$ (bottom row): for 50 pulses a) and f); 100 pulses b) and g); 300 pulses c) and h); 500 pulses d) and i); 2000 pulses e) and j).

Zuev et al. fabricated spikes on multicrystalline silicon under similar experimental conditions in a vacuum using second harmonic radiation and also observed structure growth within 50 pulses [27]. Here, the columnar submicrostructures consisted of circular tips measuring approximately $500 \mathrm{~nm}$ in diameter, column widths of $675 \mathrm{~nm}$ and a period/spacing near the wavelength, on average (Fig. 7). Similar observations resulted at $1.5 \mathrm{~J} / \mathrm{cm}^{2}$ with a loss of periodicity and resulting irregular shapes with increasing the \# of pulses (Fig. 6f-j). The geometries span columns, dendritic arrays and even conical structures with rounded tips in the micron scale.

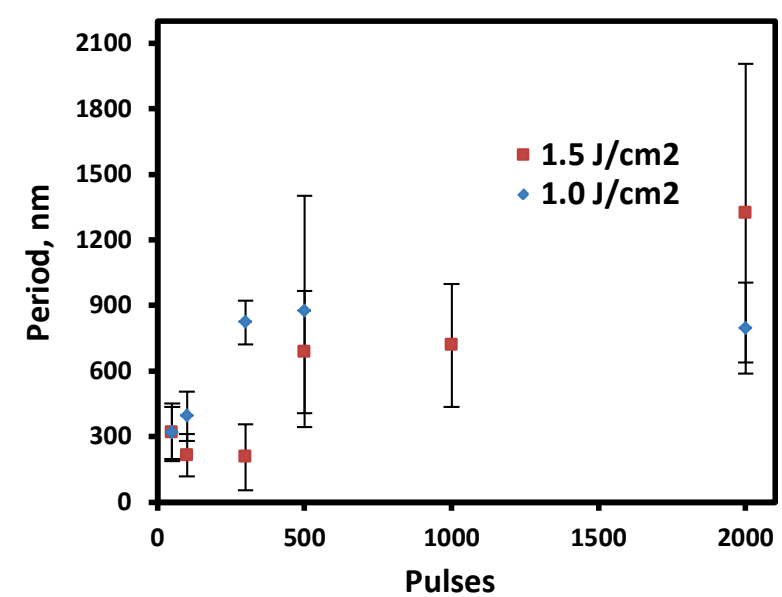

Fig. 7 Period of neighboring microstructures for 1.0 and $1.5 \mathrm{~J} / \mathrm{cm}^{2}$ for $50-2000$ pulses.

The heights of the columnar sub-microstructures were in the hundreds of nanometers to slightly above $1 \mu \mathrm{m}$, based on the magnitude of the peak heights of the structures relative to the untouched silicon surface. The height profile at the edge of the crater in the Fig 4 inset has the structure heights at $0.2 \pm 0.1 \mu \mathrm{m}$. These heights were further investigated with AFM images taken at the edge of the crater for $1.0 \mathrm{~J} / \mathrm{cm}^{2}$ and 50 pulses whereby the molten material measured several micrometers tall $(\sim 4 \mu \mathrm{m})$ and revealed the sub-microcolumn structures well below in height, again relative to the untouched Si surface (Fig 8a). The structures next to the molten material at the edge had heights of $0.5 \pm$ $0.1 \mu \mathrm{m}$ and could rise to as tall as $1.3 \mu \mathrm{m}$ (Fig 8b). The structure penetration depth measured approximately 0.3 $\mu \mathrm{m}$, giving overall structure heights to as much as $1.6 \mu \mathrm{m}$.

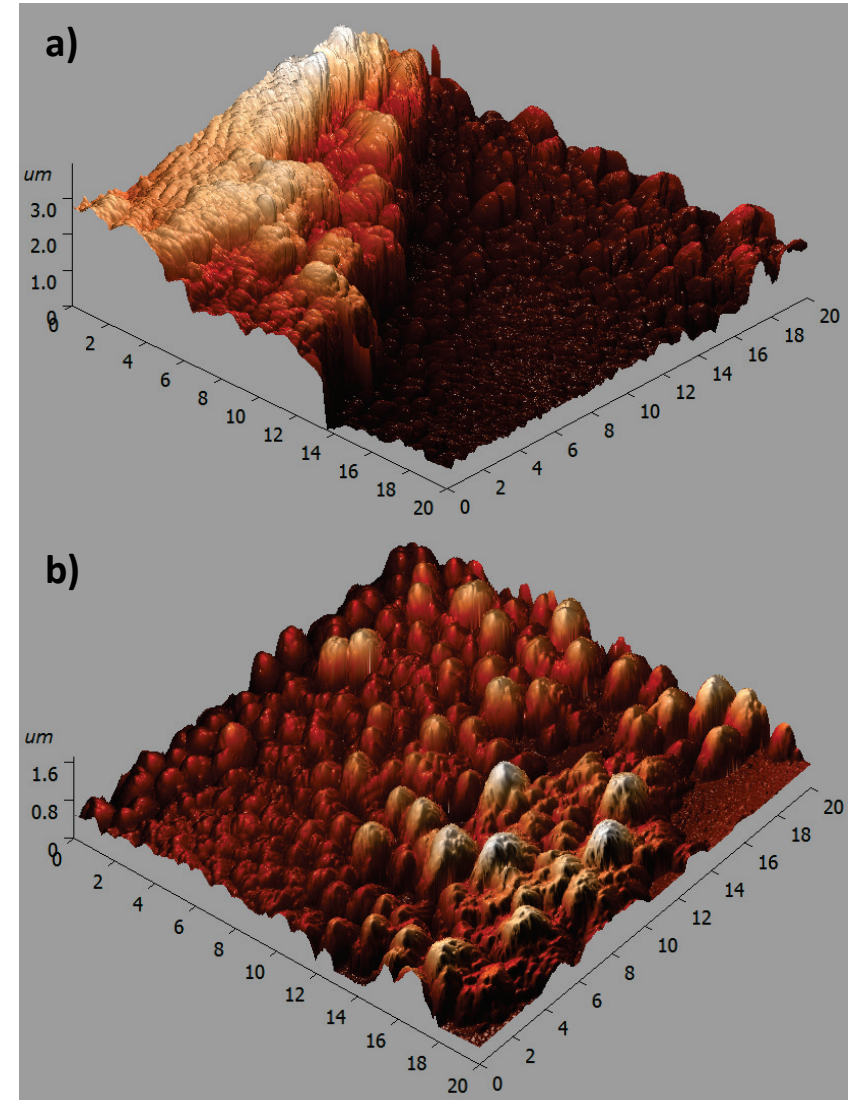

Fig. 8 AFM images taken at perimeter of crater for $1.0 \mathrm{~J} / \mathrm{cm}^{2}$ and 50 pulses: a) columnar sub-microstructures beneath and to the right of molten silicon and b) columnar sub-microstructures.

The height values for these structures are consistent with reported values of just under a micrometer [2, 6, 28]. However, others have reported heights that reached tens of microns, for both femtosecond and nanosecond laser texturing $[3,8,15,25,29,30]$.

Columnar microstructures within the ablated center were only observed sporadically at 50 and 100 pulses at select regions at $1.0 \mathrm{~J} / \mathrm{cm}^{2}$ (Fig. 9). These structures tended to line along the pushed melt fronts just below their edges [2]. This observation indicates that roughening in the nanosecond time scale is largely thermo-mechanically controlled $[14,25]$. Photothermal ablation is the primary disadvantage when texturing with nanosecond pulses because the heat-affected zones (HAZ) are significant. 


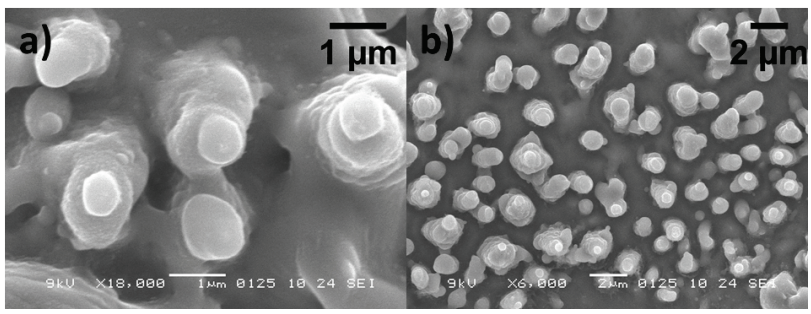

Fig. 9 Columnar microstructures at ablation centers using a fluence of $1.0 \mathrm{~J} / \mathrm{cm}^{2}$ : a) 50 pulses (scale bar: $1 \mu \mathrm{m}$ ); b) 100 pulses (scale bar: $2 \mu \mathrm{m}$ ).

In contrast, shorter pulses such as in the femtosecond time scale are less affected by HAZs, which allows for high ablating precision $[25,31]$. Crouch et al. compared nanosecond and femtosecond texturing on silicon and found larger separations for the resulting structures in the nanosecond time scale ( $8 \mu \mathrm{m}$ vs. $20 \mu \mathrm{m})$ [32]. However, texturing was performed under different experimental conditions to the ones in this contribution and results have little resemblance to the structures produced herein. A similarity to this work can be appreciated in the structure growth being above the silicon surface much like the ones presented here. In contrast, the resulting structures textured with femtosecond pulses had their tips at the surface of the silicon wafer [25, 32]. Moreover, the structures themselves are roughened when produced with femtosecond pulses and smooth for nanosecond-textured surfaces, a feature of those produced in this work [25, 32, 33]. Furthermore, an appropriate laser fluence for structuring lies between the ablation threshold and the microtexturing range reported in the literature for solid-state lasers in the nanosecond regime; however those values were not measured in this study [25].

Micro-columnar structures were observed at 2000 pulses outside of the ablation center at a laser fluence of 3 $\mathrm{J} / \mathrm{cm}^{2}$ at the focal distance (Fig. 10).

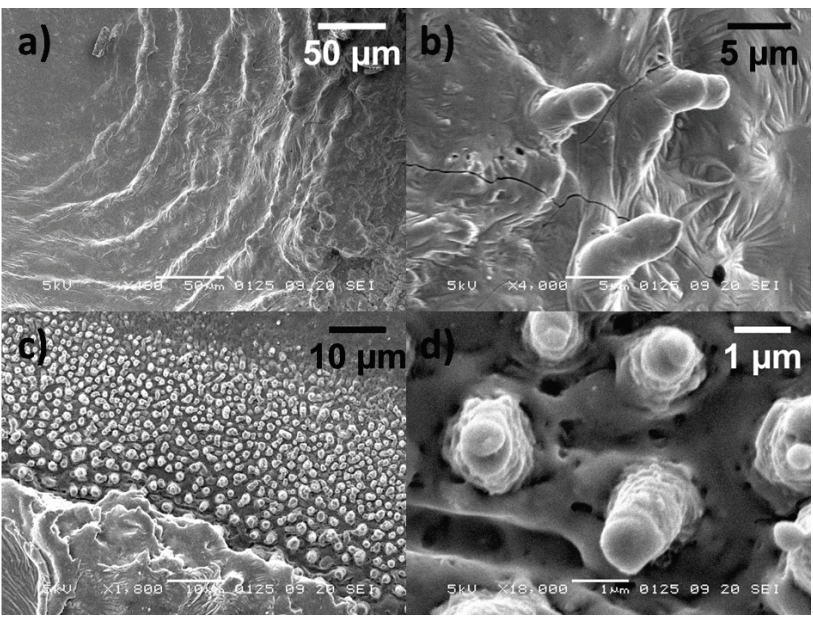

Fig. 10 Laser ablation at the lens focal point for 2000 pulses on $\mathrm{Si}$ at a fluence of $3.0 \mathrm{~J} / \mathrm{cm}^{2}$ : a) capillary waves at the ablation center; b) microstructures formed along capillary waves of the crater; c) columnar sub-microstructures formed at the border of the ablation center; d) magnified image of these structures.

These structures have a tip width of approximately $630 \mathrm{~nm}$, a column width of approximately $1.6 \mu \mathrm{m}$ and are spaced approximately $1.3 \mu \mathrm{m}$ from one another (Fig. 10d). Furthermore, this layer of microstructures expands for approximately $32 \mu \mathrm{m}$ from the ablation center (Fig. 10c). Structures formed within the craters had widths between 2.5 and $3.5 \mu \mathrm{m}$ (Fig. 10b). Capillary waves are also evident in Fig. $10 \mathrm{a}$, resulting from cooling processes as the melt is pushed towards the exterior.

The various layers of morphologies: crater formation, columnar formation, and ripple like patterns are indicative of a Gaussian energy distribution where the highest fluence is exerted at the center of the beam with decreasing energies away from the center. This also highlights the narrow fluence range permissible for structuring. In order to avoid crater formation, the sample should be moved in front of the focal point [16]. This has the added advantage of increasing the ablating area, decreasing the energy density, and using higher stabilizing lasing energies.

\section{Conclusion}

A Gaussian beam diameter was successfully measured via a knife-edge method. Columnar sub-microstructures were successfully obtained by ablating at both the focal point and in front of the focal point, predominantly at the border of the craters. The present study suggests that keeping the fluence range between 0.3 and $1.5 \mathrm{~J} / \mathrm{cm}^{2}$ and using a low number of pulses produces columnar submicrostructures in air using the third harmonic of a $\mathrm{Nd}$ :YAG pulsed nanosecond laser operating at $10 \mathrm{~Hz}$. Characterization of sub-microstructures obtained was accomplished using SEM micrographs, profilometry measurements and AFM images.

\section{Acknowledgments}

We thank Dr. Wilfredo Otaño Rivera for providing the facilities to conduct SEM experiments at the University of Puerto Rico at Cayey. Finally, we thank Mr. Adrian Camacho-Berrios for assisting in the profilometry experiments and the collection of SEM micrographs.

Support from the U.S. Department of Homeland Security under Award Number 2013-ST-061-ED0001 is also acknowledged. However, the views and conclusions contained in this document are those of the authors and should not be considered as a representation of the official policies, either expressed or implied, of the U.S. Department of Homeland Security.

This material is based upon work supported in part by the U. S. Army Research Laboratory and the U. S. Army Research Office under contract/grant number W911NF1410414."

\section{References}

[1] M. Shen, J.E. Carey, C.H. Crouch, M. Kandyla, H.A. Stone, and E. Mazur: Nano Lett., 8, (2008) 2087.

[2] B.K. Nayak, K. Sun, C. Rothenbach, and M.C. Gupta: Appl. Opt., 50, (2011) 2349.

[3] B.K. Nayak and M.C. Gupta: Appl. Opt., 51, (2012) 114.

[4] J.A. Stolee, Y. Chen, and A. Vertes: J. Phys. Chem. C, 114, (2010) 5574.

[5] S.C. Bayliss, L.D. Buckberry, I. Fletcher, and M.J. Tobin: Sens. Actuators A Phys., 74, (1999) 139.

[6] Y. Chen and A. Vertes: Anal. Chem., 78, (2006) 5835. 
[7] E.D. Diebold, N.H. Mack, S.K. Doorn, and E. Mazur: Langmuir, 25, (2009) 1790.

[8] B.R. Tull, J.E. Carey, E. Mazur, J.P. McDonald, and S.M. Yalisove: MRS Bull., 31, (2006) 626.

[9] D. Batani: "Laser Ablation and Laser Induced Plasmas for Nanomachining and Material Analysis," ed. by A. Vaseashta and I.N. Mihailescu, (Springer, Dordrecht, 2008) p.165.

[10] M. Bussoli, D. Batani, T. Desai, F. Canova, M. Milani, M. Trtica, B. Gakovic, and E. Krousky: Laser Part. Beams, 25, (2007) 121.

[11] M. Brown and C. Arnold: "Fundamentals of LaserMaterial Interaction and Application to Multiscale Surface Modification," ed. by K. Sugioka, M. Meunier, and A. Piqué, (Springer-Verlag, Berlin Heidelberg, 2010) p.91.

[12] S.I. Dolgaev, S.V. Lavrishev, A.A. Lyalin, A.V. Simakin, V.V. Voronov, and G.A. Shafeev: Appl. Phys. A Mater. Sci., 73, (2001) 177.

[13] S.R. Foltyn: "Surface modification of materials by cumulative laser irradiation," in "Pulsed laser deposition of thin films," ed. by D.B. Chrisey and G.K. Hubler, (J. Wiley, New York, 1994) p.89.

[14] D. Bäuerle: "Laser Processing and Chemistry," (Springer-Verlag, Berlin Heidelberg, 2011) p. 623.

[15] A.J. Pedraza, J.D. Fowlkes, and D.H. Lowndes: Appl. Phys. Lett., 74, (1999) 2322.

[16] M.Y. Shen, C.H. Crouch, J.E. Carey, and E. Mazur: Appl. Phys. Lett., 85, (2004) 5694.

[17] M.A. de Araújo, R. Silva, E. de Lima, D.P. Pereira, and P.C. de Oliveira: Appl. Opt., 48, (2009) 393.

[18] M. Mauck: Appl. Opt., 18, (1979) 599.

[19] D.K. Cohen, B. Little, and F.S. Luecke: Appl. Opt., 23, (1984) 637.

[20] Y. Suzaki and A. Tachibana: Appl. Opt., 14, (1975) 2809.
[21] J.A. Arnaud, W.M. Hubbard, G.D. Mandeville, B. de la Clavière, E.A. Franke, and J.M. Franke: Appl. Opt., 10, (1971) 2775.

[22] W.H. Press, B.P. Flannery, S.A. Teukolsky, and W.T. Vetterling: "Numerical recipes in $\mathrm{C}$ : the art of scientific computing," (Cambridge University Press, Cambridge, 1992) p.650.

[23] A. Savitzky and M.J.E. Golay: Anal. Chem., 36, (1964) 1627.

[24] B. Farkas and Z. Geretovszky: Appl. Surf. Sci., 252, (2006) 4728.

[25] M. Gupta: J. Laser Micro Nanoen., 8, (2013) 124.

[26] E. Hecht: "Optics," (Addison Wesley, San Francisco, 2002) p.594.

[27] D. Zuev, O. Novodvorsky, E. Khaydukov, O. Khramova, A. Lotin, L. Parshina, V. Rocheva, V. Panchenko, V. Dvorkin, A. Poroykov, G. Untila, A. Chebotareva, T. Kost, and M. Timofeyev: Appl. Phys. B, 105, (2011) 545 .

[28] J.P. Moening and D.G. Georgiev: J. Appl. Phys., 107, (2010) 014307.

[29] C. Wu, C.H. Crouch, L. Zhao, J.E. Carey, R. Younkin, J.A. Levinson, E. Mazur, R.M. Farrell, P. Gothoskar, and A. Karger: Appl. Phys. Lett., 78, (2001) 1850.

[30] D.H. Lowndes, J.D. Fowlkes, and A.J. Pedraza: Appl. Surf. Sci., 154-155, (2000) 647.

[31] K. Ahmmed, C. Grambow, and A.-M. Kietzig: Micromachines, 5, (2014) 1219.

[32] C.H. Crouch, J.E. Carey, J.M. Warrender, M.J. Aziz, E. Mazur, and F.Y. Génin: Appl. Phys. Lett., 84, (2004) 1850.

[33] A.Y. Vorobyev and C. Guo: Laser Photon. Rev., 7, (2013) 385.

(Received: March 26, 2015, Accepted: June 8, 2015) 